\title{
Unitary Holonomies by Direct Degenerate Projections
}

\author{
Daniel K. L. $\mathrm{Oi}^{1, *}$ \\ ${ }^{1}$ SUPA Department of Physics, University of Strathclyde, Glasgow G4 ONG, United Kingdom
}

(Dated: May 7, 2014)

\begin{abstract}
An incomplete quantum measurement can induce non-trivial dynamics between degenerate subspaces, a closed sequence of such projections produces a non-abelian holonomy. We show how to induce unitary evolution on an initial subspace from such finite discrete sequences and also construct a near deterministic repeat-until-success protocol. We also prove necessary and sufficient criteria on the auxiliary Hilbert space dimension required for inducing isometries between between subspaces.
\end{abstract}

PACS numbers: 03.65.Aa,03.65.Vf,03.67.-a

The geometric structure of quantum theory is highlighted by the phenomenon of the Pancharatnam-Berry Phase whereby the cyclic evolution of a pure quantum state induces a geometric phase $(U(1)$ abelian holonomy) in addition to the standard dynamic phase [1, 2]. Nonabelian holonomies can be induced by the cyclic adiabatic modulation of a Hamiltonian with a degenerate subspace [3] or by non-adiabatic means [4]. Alternatively, the evolution of a subspace can be driven deterministically by a dense sequence of incomplete (degenerate) projections again leading to a holonomy [5]. Such Zeno effects have been proposed for quantum control and computation [6, 7], and for engineered quantum systems [8].

The more practical case of finite projective sequences was addressed by Anandan and Pines [5] and later by Åberg, Kult, and Sjöqvist [9, 10] where they analysed the geometric structure of sequences of points in the Stiefel manifold of projective subspaces, and found the associated holonomies. Here, we extend this by explicitly constructing finite discrete sequences of degenerate projections that induce isometries between subspaces and demonstrate two methods of achieving unitary holonomic evolution. The first is minimal in that only one auxiliary Hilbert space dimension is required, though at the expense of the success probability. The second provides a near deterministic protocol, but requires a doubling of the Hilbert space dimension. We also prove that this doubling is a necessary condition for stepwise unitary (isometric) subspace dynamics.

In an $N$-dimensional Hilbert space we can perform an incomplete measurement where one outcome is a degenerate projection onto a $k$-dimensional subspace and the complementary result can be taken as a projection onto a $N-k$ dimensional subspace. Without loss of generality we will identify a projection operator with its +1 eigenspace or a set of basis vectors. Consider an initial state $\left|\psi_{0}\right\rangle$ lying in a $k$-dimensional subspace associated with a projector $\Pi_{0}$. Applying a second $k$-dimensional projector $\Pi_{1}$ (assumed to be non-orthogonal to $\Pi_{0}$ ), the system survives with probability $p_{1}=\left\langle\psi_{0}\left|\Pi_{1}\right| \psi_{0}\right\rangle$ and now lies within the subspace of $\Pi_{1}$. The normalized conditional state is given by $\Pi_{1}\left|\psi_{0}\right\rangle / \sqrt{p_{1}}$.
Extending this to a sequence of projections $\left\{\Pi_{j}\right\}_{j=0}^{n}$ where the final projection $\Pi_{n}$ coincides with $\Pi_{0}$, the system may undergo a cyclic evolution and return to its original subspace. The final conditional state is related to the initial state by

$$
\left|\psi_{f}\right\rangle=\Gamma\left|\psi_{0}\right\rangle / \sqrt{p_{f}}
$$

where the cumulative operation is given by

$$
\Gamma=\prod_{j} \Pi_{j}
$$

and the survival probability is $p_{f}=\left\langle\psi_{0}\left|\Gamma^{\dagger} \Gamma\right| \psi_{0}\right\rangle$. In general, $\Gamma$ is not proportional to a unitary operation on the initial subspace. In the limit of a dense sequence of projections approaching a continuous path in the associated Grassmann manifold, then $\Gamma$ becomes unitary [9].

We require only one additional dimension $(N=k+1)$ in order to generate a unitarily proportional $\Gamma$ using a finite sequence. To illustrate, we construct a unitary operation $U=\sum e^{i \phi_{m}}\left|\psi_{m}\right\rangle\left\langle\psi_{m}\right|$ chosen to be diagonal in some orthonormal basis $\left\{\left|\psi_{m}\right\rangle\right\}_{m=1}^{k}$ for the initial subspace. The process proceeds stepwise by generating each phase factor in turn by a sub-sequence of projections driving the $m^{\text {th }}$ component of the superposition around a loop within a two-dimensional subspace spanned by $\left|\psi_{m}\right\rangle$ and a single auxiliary level $\left|\psi_{a}\right\rangle$. Each loop generates a geometric phase equal to half of the solid angle enclosed on the Bloch sphere.

Specifically, to generate $\phi_{m}$ we use a sub-sequence $\left\{\Pi_{m}^{l}\right\}_{l=0}^{N_{m}}$ where the starting and ending subspaces coincides with $\Pi_{0}$, i.e. $\Pi_{m}^{0}=\Pi_{m}^{N_{m}}=\Pi_{0}$,

$$
\Pi_{m}^{l}=\left(\sum_{j \neq m}\left|\psi_{j}\right\rangle\left\langle\psi_{j}\right|\right)+\left|\psi_{m}^{l}\right\rangle\left\langle\psi_{m}^{l}\right|,
$$

with $\left|\psi_{m}^{0}\right\rangle=\left|\psi_{m}^{N_{m}}\right\rangle=\left|\psi_{m}\right\rangle$, and $\left|\psi_{m}^{l}\right\rangle$ traces a path in the the subspace of $\left\{\left|\psi_{m}\right\rangle,\left|\psi_{a}\right\rangle\right\}$. The first term on the right hand side simply projects onto all but one of the spanning basis vectors on $\Pi_{0}$. The cyclic evolution of the remaining term is responsible for generating the $\phi_{m}$-phase (Fig. 1). An initial state $|\psi\rangle=\sum \alpha_{j}\left|\psi_{j}\right\rangle$ in the image 


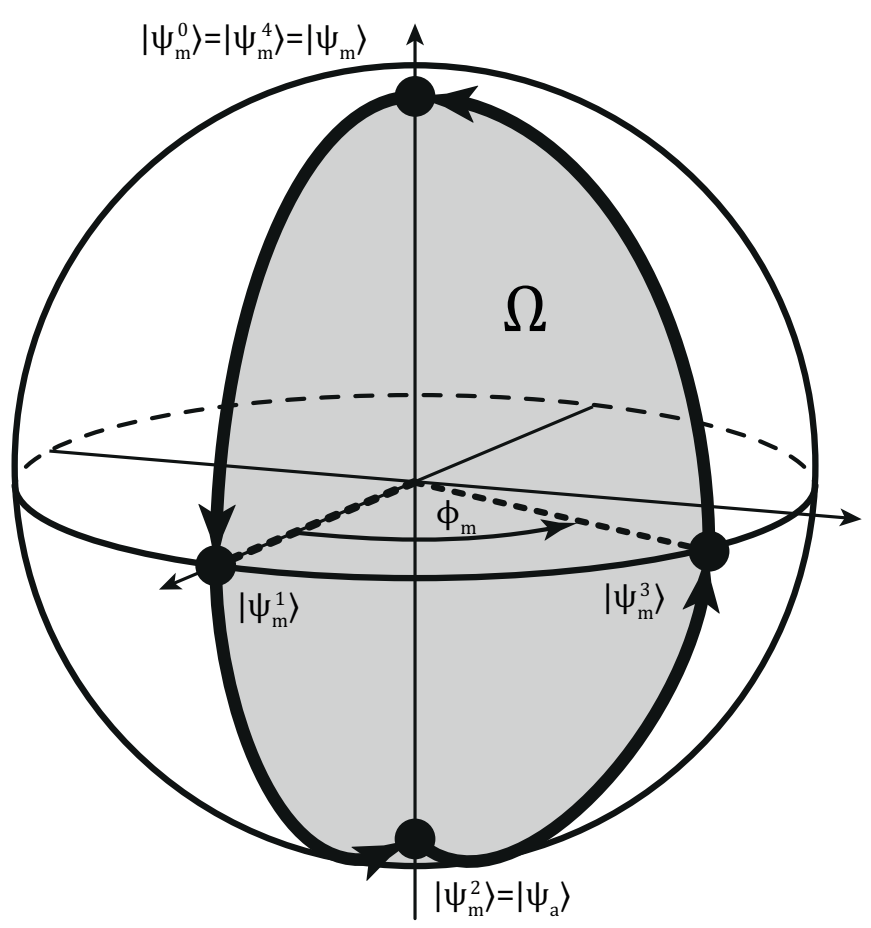

FIG. 1. Geometric phase of the $\left|\psi_{m}\right\rangle$ component. The $\left|\psi_{m}\right\rangle$ component of the $\Pi_{0}$ subspace is driven by a projective subsequence along the closed path indicated by the thick arrows within a two dimensional subspace represented by a Bloch sphere. The transition amplitudes are equal in magnitude between the four points (solid circles). The Pancharatnam or geometric phase associated with the cyclic evolution is half of the solid angle $\Omega$ enclosed (shaded) and is equal to $\phi_{m}$.

of $\Pi_{0}$ will transform under the sequence $\Gamma_{m}=\prod_{l} \Pi_{m}^{l}$ into the unnormalized state $\sum_{j \neq m} \alpha_{j}\left|\psi_{j}\right\rangle+t_{m} \alpha_{m}\left|\psi_{m}\right\rangle$ where $t_{m}=\operatorname{Tr}\left[\prod_{l=0}^{N_{m}}\left|\psi_{m}^{l}\right\rangle\left\langle\psi_{m}^{l}\right|\right]$ and $\arg t_{m}=\phi_{m}$. We require that $\left|t_{m}\right|>0$ for a non-trivial success probability. A possible subsequence $\Pi_{m}^{l}$ can be specified by

$$
\begin{aligned}
\left|\psi_{m}^{0}\right\rangle & =\left|\psi_{m}^{4}\right\rangle=\left|\psi_{m}\right\rangle, \quad\left|\psi_{m}^{2}\right\rangle=\left|\psi_{a}\right\rangle, \\
\left|\psi_{m}^{1}\right\rangle & =\frac{1}{\sqrt{2}}\left(\left|\psi_{m}\right\rangle+\left|\psi_{a}\right\rangle\right), \\
\left|\psi_{m}^{3}\right\rangle & =\frac{1}{\sqrt{2}}\left(\left|\psi_{m}\right\rangle+e^{i \phi_{m}}\left|\psi_{a}\right\rangle\right),
\end{aligned}
$$

with $\left|t_{m}\right|^{2}=\left(\frac{1}{2}\right)^{4}=\frac{1}{16}$. Increasing $N_{m}$ would enable the transition probability to increase until in the limit of $N_{m} \rightarrow \infty$ we induce the Zeno effect and $t_{m} \rightarrow 1$.

Applying $\Gamma_{m}$ for each $m$ leads to the final unnormalized state

$$
\left|\psi^{\prime}\right\rangle=\sum_{m} t_{m} \alpha_{m}\left|\psi_{m}\right\rangle=\Gamma|\psi\rangle
$$

where $\Gamma=\prod_{m} \Gamma_{m}=\sum t_{m}\left|\psi_{m}\right\rangle\left\langle\psi_{m}\right|$. For the conditional operation to be unitary on the initial subspace, all the amplitudes should be reduced by the same factor so that the survival probability is independent of the initial state, i.e. $\left|t_{m}\right|=t$. This can be ensured either through suitable choice of $\Gamma_{m}$, or by a final filtering operation to equalize $t_{m}$ to their smallest magnitude. The success probability of any initial state is then $t^{2}$ and the conditional evolution is unitary as required, $\left|\psi^{\prime}\right\rangle / \|\left|\psi^{\prime}\right\rangle \|=(\Gamma / t)|\psi\rangle=U|\psi\rangle$.

The procedure above creates a final unitary operation from the conditional success of several non-unitary steps. Any information gain at each step is offset in subsequent projections so overall no information is gained about $|\psi\rangle$ conditional on all steps succeeding. An alternate procedure would ensure that every transition is an isometry between the source and image subspaces and this requirement leads to a restriction on the minimum dimension $N$ of the embedding Hilbert space, as shown below.

Let $\Pi_{0}$ and $\Pi_{1}$ be nonorthogonal $k$-degenerate projectors specified with orthonormal bases $\mathcal{B}_{0}=\left\{\left|\mu_{j}\right\rangle\right\}_{j=1}^{k}$ and $\mathcal{B}_{1}=\left\{\left|\nu_{j}\right\rangle\right\}_{j=1}^{k}$ respectively. Let $\operatorname{span}\left(\mathcal{B}_{0} \cup \mathcal{B}_{1}\right)$ be $\left(k+k^{\prime}\right)$-dimensional, $1 \leq k^{\prime} \leq k$. We can augment $\mathcal{B}_{0}$ with $k^{\prime}$ extra vectors $\left\{\left|\mu_{j}\right\rangle\right\}_{j=k+1}^{k+k^{\prime}}$ to form a basis $\mathcal{B}_{0}^{\prime}=\left\{\left|\mu_{j}\right\rangle\right\}_{j=1}^{k+k^{\prime}}$ for the combined subspace. We now use the augmented basis $\mathcal{B}_{0}^{\prime}$ to express the vectors of $\mathcal{B}_{1}$ as $\left\{\left|\nu_{j^{\prime}}\right\rangle=\sum_{j=1}^{k+k^{\prime}} C_{j^{\prime} j}\left|\mu_{j}\right\rangle\right\}_{j^{\prime}=1}^{k}$, where $C$ is a $k \times\left(k+k^{\prime}\right)$ complex matrix with orthonormal rows. Using Gaussian elimination on $C$, we can find a matrix $D$ which defines a new orthonormal basis for $\Pi_{1}, \mathcal{B}_{1}^{\prime}=\left\{\left|\nu_{j^{\prime}}^{\prime}\right\rangle=\right.$ $\left.\sum_{j=1}^{k+k^{\prime}} D_{j^{\prime} j}\left|\mu_{j}\right\rangle\right\}_{j^{\prime}=1}^{k}$, where $\left\{\left|\nu_{j^{\prime}}^{\prime}\right\rangle\right\}_{j^{\prime}=1}^{k-k^{\prime}} \subset \operatorname{span}\left(\mathcal{B}_{0}\right)$, and only $\left\{\left|\nu_{j^{\prime}}^{\prime}\right\rangle\right\}_{l^{\prime}=k-k^{\prime}+1}^{k}$ have support outside of $\operatorname{span}\left(\mathcal{B}_{0}\right)$. This implies that $\Pi_{0}$ and $\Pi_{1}$ share a $\left(k-k^{\prime}\right)$ dimensional subspace. The $k^{\prime}$ elements of $\mathcal{B}_{1}^{\prime}$ not in this common subspace can be written, up to a relative phase, as $\left|\nu_{j^{\prime}}^{\prime}\right\rangle=\cos \theta_{j^{\prime}}\left|m_{j^{\prime}}\right\rangle+\sin \theta_{j^{\prime}}\left|n_{j^{\prime}}\right\rangle$, where $\left|m_{j^{\prime}}\right\rangle \in \operatorname{span}\left(\mathcal{B}_{0}\right)$, $\left|n_{j^{\prime}}\right\rangle \in \operatorname{ker}\left(\Pi_{0}\right)$, and $0<\theta_{j^{\prime}}<\pi / 2$.

If $\Pi_{1}$ induces a non-trivial isometry on $\operatorname{span}\left(\mathcal{B}_{0}\right)$, this implies that $\Pi_{0}$ and $\Pi_{1}$ do not share any common nontrivial eigenvectors, i.e. $k=k^{\prime}$, otherwise the transition probabilities for states in the common eigenspace and those with outside support would differ. Hence we can express $\mathcal{B}_{1}^{\prime}=\left\{\cos \theta\left|m_{j^{\prime}}\right\rangle+\sin \theta\left|n_{j^{\prime}}\right\rangle\right\}_{j^{\prime}=1}^{k}$, and the transition probability between the subspaces is $t^{2}=\cos ^{2} \theta$. Completing the resolution of the identity specifies a measurement, and the complementary outcome to $\Pi_{1}$ given by $\widetilde{\Pi}_{1}=\mathbb{I}-\Pi_{1}$ is also a projective isometry that translates the subspace of $\Pi_{0}$ to one that is orthogonal to $\Pi_{1}$. If we make a two-outcome measurement $\left\{\Pi_{1}, \widetilde{\Pi}_{1}\right\}$, regardless of the resulting projection the information originally in the subspace of $\Pi_{0}$ is preserved. We now use this to construct a repeat-until-success protocol.

We illustrate the basic idea by implementing isometries on a qubit initially embedded in the $\{|0\rangle,|1\rangle\}$ subspace. We augment the system by the direct sum of 


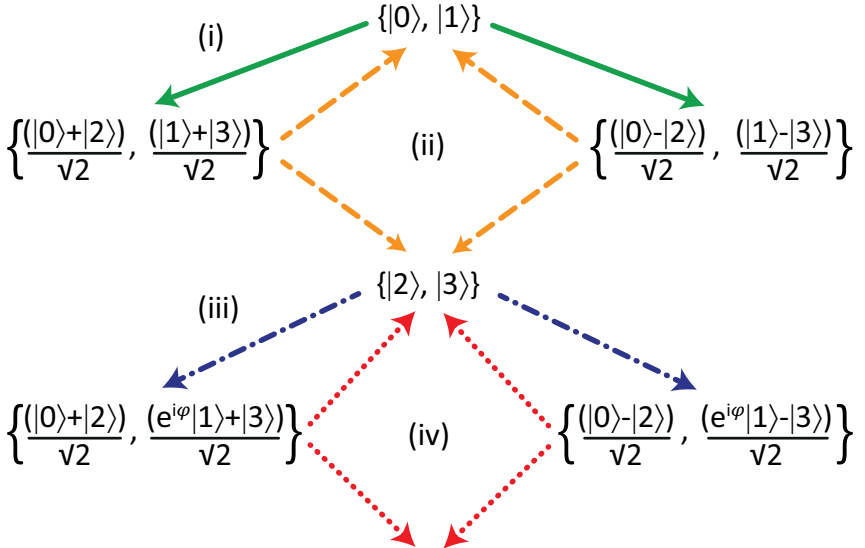

$\{|0\rangle,|1\rangle\}$

FIG. 2. (Color online) Qubit unitary projection sequence. Each two-outcome measurement is indicated by a pair of similar arrows between nodes representing the initial and final subspaces associated with degenerate projections. (i) The qubit is initially in the $\{|0\rangle,|1\rangle\}$ subspace and the first measurement (solid green lines) projects equiprobably onto the $\{(|0\rangle+|2\rangle) / \sqrt{2},(|1\rangle+|3\rangle) / \sqrt{2}\}$ subspace or its complement, $\{(|0\rangle-|2\rangle) / \sqrt{2},(|1\rangle-|3\rangle) / \sqrt{2}\}$. (ii) The second measurement (dashed yellow) projects back onto the original $\{|0\rangle,|1\rangle\}$ subspace or forwards onto the $\{|2\rangle,|3\rangle\}$ subspace that acts as a "checkoint". In the former case, the qubit is unaltered and we begin again. (iii) For the $\{|2\rangle,|3\rangle\}$ outcome, the next (third) measurement (dash-dotted blue) begins the return loop via a different subspace. (iv) The fourth measurement (dotted red) either projects back to the $\{|2\rangle,|3\rangle\}$ checkpoint subspace or completes the loop returning the system to the initial subspace. The qubit will have undergone a unitary holonomy $U=e^{i \frac{\varphi}{2}} e^{-i \varphi \frac{\sigma z}{2}}$, up to a \pm 1 global phase factor depending on the path taken through the graph. The rotation axis of $U$ can be chosen by redefinition of $\{|0\rangle,|1\rangle\}$. As each transition is equally weighted $\left(p=\frac{1}{2}\right)$, the mean time for traversing the graph top to bottom can be shown to be 8 steps.

two additional levels, $\{|2\rangle,|3\rangle\}$ and define a measurement with two 2-dimensional degenerate projections with subspaces,

$$
\begin{aligned}
& \Pi_{1}:\left\{\cos \theta|0\rangle+e^{i \phi} \sin \theta|2\rangle, \cos \theta|1\rangle+e^{i \varphi} \sin \theta|3\rangle\right\} \\
& \widetilde{\Pi}_{1}:\left\{\sin \theta|0\rangle-e^{i \phi} \cos \theta|2\rangle, \sin \theta|1\rangle-e^{i \varphi} \cos \theta|3\rangle\right\},
\end{aligned}
$$

where the phases $\{\phi, \varphi\}$ are arbitrary. The measurement probabilities are independent of the initial state and are $P_{1}=\cos ^{2} \theta$ and $P_{\tilde{1}}=\sin ^{2} \theta$ respectively. Conditional on the result, we can choose different pairs of degenerate projections to measure in the next round, each subsequent measurement may depend on previous outcomes resulting in a binary tree of projections [11]. If at some point in the sequence the resultant subspace returns to the original one, a unitary holonomy would be the result. A randomly choosen sequence of such measurements periodically revisiting the original subspace can generate a desired unitary in an expected number of steps polynomial in the approximation error [12-14].
Alternatively, it may be more efficient for the projection sequence to trace out specific paths, Fig. 2 demonstrates such a sequence that implements a near deterministic qubit rotation. An initial qubit state is translated between different subspaces according to the directed graph structure until it returns to the original subspace having had applied to it a unitary holonomy. Due to measurement randomness, the measurements may need to be repeated until a successful sequence of results is obtained. The probability of failure decreases exponentially in the total number of allowed steps and repeatuntil-success protocols have been used to good effect in gate synthesis [15].

As a concrete example, we implement $U=e^{i \frac{\varphi}{2}} e^{-i \varphi \frac{\sigma_{z}}{2}}$ on an initial state $|\psi\rangle=\alpha|0\rangle+\beta|1\rangle$ assuming that we take the minimal traversal (no backtracking) down the right side of the graph in Fig. 2. The first measurement takes us to the subspace $\{(|0\rangle-|2\rangle) / \sqrt{2},(|1\rangle-|3\rangle) / \sqrt{2}\}$ and the system becomes

$$
\begin{aligned}
\left|\psi_{1}\right\rangle & =\frac{\left(\frac{|0\rangle-|2\rangle}{\sqrt{2}} \frac{\langle 0|-\langle 2|}{\sqrt{2}}+\frac{|1\rangle-|3\rangle}{\sqrt{2}} \frac{\langle 1|-\langle 3|}{\sqrt{2}}\right)}{\sqrt{\frac{1}{2}}}|\psi\rangle \\
& =\alpha \frac{|0\rangle-|2\rangle}{\sqrt{2}}+\beta \frac{|1\rangle-|3\rangle}{\sqrt{2}},
\end{aligned}
$$

where in the first line we have normalized the state by the square root of the transition probability which is independent of $|\psi\rangle$ as required by isometry. If the second projection does not take us back up to the original subspace but down to the next "checkpoint" subspace $\{|2\rangle,|3\rangle\}$ instead, the state is now

$$
\left|\psi_{2}\right\rangle=-(\alpha|2\rangle+\beta|3\rangle) .
$$

The third measurement begins the return loop but via a different subspace, an outcome to the right results in

$$
\left|\psi_{3}\right\rangle=\alpha \frac{|0\rangle-|2\rangle}{\sqrt{2}}+\beta \frac{e^{i \varphi}|1\rangle-|3\rangle}{\sqrt{2}} .
$$

A final successful measurement completes the loop leaving the qubit back in its original subspace,

$$
\left|\psi_{4}\right\rangle=\alpha|0\rangle+e^{i \varphi} \beta|1\rangle=U|\psi\rangle
$$

as required. A simple calculation shows that holonomies corresponding to different traversals only differ by a \pm 1 global phase factor.

We can generalize the procedure in Fig. 2 to induce a $k$-dimensional unitary on an initial subspace spanned by $\{|j\rangle\}_{j=1}^{k}$. We augment the Hilbert space with an additional $k^{\prime}=k$ levels $\{|\bar{j}\rangle\}_{j}^{k}$. We now project onto subspaces spanned by (unnormalized) vectors $\{|j\rangle+|\bar{j}\rangle\}$ and $\{|j\rangle-|\bar{j}\rangle\}$ for the first measurement, $\{|j\rangle\}$ and $\{|\bar{j}\rangle\}$ for the second measurements, $\left\{e^{i \phi_{j}}|j\rangle+|\bar{j}\rangle\right\}$ and $\left\{e^{i \phi_{j}}|j\rangle-|\bar{j}\rangle\right\}$ for the third measurements, and $\{|j\rangle\}$ and 
$\{|\bar{j}\rangle\}$ for the fourth and final measurements in the graph. The induced holonomy after a successful sequence of projections is given by $U=\operatorname{diag}\left(e^{i \phi_{m}}\right)$ in the $\{|j\rangle\}$ basis, up to a \pm 1 global phase. The graph structure is identical to that in Fig. 2 with the same transition probabilities and expected transit time of 8 steps.

In summary we have elucidated criteria and restrictions for inducing isometries between subspaces by discrete projections, complementing previous work exploring the Zeno regime $[6,7]$ or formal aspects of projective holonomies $[5,9,10]$. In order to preserve information during each projection, the dimensionality of the entire space must be at least twice that of the initial subspace. The direct and iterative holonomies coincide in this case [10]. Using a cyclic sequence of projections we construct a repeat-until-success protocol to implement a general unitary operation with an average of 8 measurements. If doubling the initial subspace dimension is not possible, we also show that a single additional level is sufficient for inducing a unitary holonomy. In this case, the trade-off is in the success probability, though it can be increased with more projections until we ultimately recover the Zeno effect in the infinite limit.

The required highly degenerate projections may be possible experimentally. The proposal in [16] suggests a way of performing infinitely degenerate projections on photon number in cavity quantum electrodynamics with displacements and squeezing to effectively modify the projection subspace. An intriguing possibility in such infinite dimensional systems is the creation of additional empty levels, as in the Hilbert Hotel Paradox [17, 18], to act as ancillary dimensions as required for stepwise isometries. This may require the development of more non-Gaussian operations in order to perform the required manipulations of the states to project onto different subspaces in conjunction with the techniques outlined in [16].

Comparing this work with measurement based quantum computation (MBQC) [19] and ancilla driven computation (ADQC) [20-22] which also employ measurement to drive dynamics, the key differences are that in the latter two schemes, a tensor product structure is assumed and measurement is performed on subsystems, not subspaces $[11,23]$. Our results are more general since a tensor product space can be mapped to a direct sum, but not neccessarily the converse. The minimal addition of a single qubit (e.g. in ADQC) automatically doubles the dimensionality and this doubling is both necessary and sufficient for unitary conditional evolution via projections on the qubit. In some experimental implementations, e.g. using position degrees of freedom [24], it is comparatively easy to increase dimensionality by the direct sum of ancillary levels, rather than add subsystems and couple them to perform entangling operations.

We finally note that near deterministic unitary holonomies require that coherence is preserved at each step. The results of [25] and references therein show that it is impossible, with unit probability, to "unlearn" information gained from a measurement outcome whose Kraus operator does not have a flat distribution of singular values. Hence this rules out the possibility of measurement trees or graphs where all final cumulative results are unitary but for which some of the intermediate effects are not isometries. The two classes of protocol presented lie at the ends of the spectrum, either preserving coherence at every step, or else only one of the final outcomes is unitary with the rest collapsing the state.

DKLO thanks Si-Hui Tan, Kuldip Singh, John Jeffers, Václav Potoček, Kerem Halil-Shah, and Johan Åberg for comments and useful discussions, and is supported by Quantum Information Scotland (QUISCO).

*daniel.oi@strath.ac.uk

[1] S. Pancharatnam, Proc. Ind. Acad. Sci. A 44, 247 (1956)

[2] M. V. Berry, Proc. R. Soc. London A 392, 45 (1984)

[3] F. Wilczek and A. Zee, Phys. Rev. Lett. 52, 2111 (1984)

[4] J. Anandan, Phys. Lett. A 133, 171 (1988)

[5] J. Anandan and A. Pines, Phys. Lett. A 141, 335 (1989)

[6] D. Burgarth, et al., Phys. Rev. A 88, 042107 (2013)

[7] J. M. Dominy, G. A. Paz-Silva, A. T. Rezakhani and D. A. Lidar, J. Phys. A: Math. Theor. 46, 075306 (2013)

[8] J.-M. Raimond et al., Phys. Rev. A 86, 032120 (2012)

[9] D. Kult, J. Åberg, E. Sjöqvist, Phys. Rev A 74, 022106 (2006)

[10] E. Sjöqvist, D. Kult, J. Åberg, Phys. Rev. A 74, 062101 (2006)

[11] E. Andersson and D. K. L Oi, Physical Review A 77, 052104 (2008)

[12] K. Halil-Shah, D. K. L. Oi, Proc. TQC 2013, LIPIcs 22, 1 (2013)

[13] K. Halil-Shah, D. K. L. Oi, arXiv:1311.3463 (2013)

[14] K. Halil-Shah, D. K. L. Oi, arXiv:1401.8004 (2014)

[15] A. Paetznick, K. M. Svore, arXiv:1311.1074 (2013)

[16] D. K. L. Oi, V. Potoček, and J. Jeffers, Phys. Rev. Lett. 110, 210504 (2013)

[17] H. Hermes, W. Markwald, "Foundations of mathematics" H. Behnke (ed.) et al. (ed.), Fundamentals of Mathematics 1, p.3, MIT (1986) (Edition: Third)

[18] V. Potoček, D. K. L. Oi, J. Jeffers, In preparation (2014)

[19] R. Raussendorf, D. E. Browne and H. J. Briegel, Phys. Rev. A 68, 022312 (2003)

[20] E. Kashefi, et al., Electronic Notes in Theoretical Computer Science 249, 307-331 (2009)

[21] J. Anders, et al., Physical Review A 82, 020301(R) (2010)

[22] J. Anders, et al., Theoretical Computer Science 430, 51 (2012)

[23] Guoming Wang and Mingsheng Ying, arXiv:quant$\mathrm{ph} / 0608235$

[24] S. G. Schirmer, D. K. L. Oi, A. D. Greentree, Phys. Rev. A 71, 012325 (2005)

[25] D. K. L. Oi, arXiv:1404.3784 (2014) 